\title{
Accuracy of laser speckle contrast imaging in the assessment of pediatric scald wounds
}

Robin Mirdell, Simon Farnebo, Folke Sjöberg and Erik Tesselaar

The self-archived postprint version of this journal article is available at Linköping University Institutional Repository (DiVA):

http:// urn.kb.se/ resolve?urn=urn:nbn:se:liu:diva-144874

N.B.: When citing this work, cite the original publication.

Mirdell, R., Farnebo, S., Sjöberg, F., Tesselaar, E., (2018), Accuracy of laser speckle contrast imaging in the assessment of pediatric scald wounds, Burns, 44(1), 90-98.

https://doi.org/ 10.1016/j.burns.2017.06.010

Original publication available at:

https:// doi.org/ 10.1016/j.burns.2017.06.010

Copyright: Elsevier

http:// www.elsevier.com/

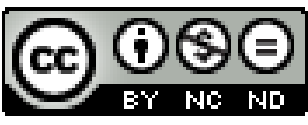




\section{Accuracy of Laser Speckle Contrast Imaging in the assessment of pediatric scald wounds}

\section{Robin Mirdell' ${ }^{1}$, Simon Farnebo ${ }^{1,3}$, Folke Sjöberg1,3, Erik Tesselaar ${ }^{1,2}$.}

1. Department of Clinical and Experimental Medicine, Faculty of Health Sciences, Linköping University, Linköping, Sweden

2. Department of Radiation Physics, Department of Medical and Health Sciences, Linköping University, Linköping, Sweden.

3. Department of Plastic Surgery, Hand Surgery, and Burns, Linköping University, Linköping, Sweden

Corresponding author:

Robin Mirdell

Department of Clinical and Experimental Medicine

Faculty of Health Sciences

Linköping University

SE-58185 Linköping, Sweden

E-mail: robin.mirdell@liu.se

Keywords: burn severity, burn assessment, scalds, laser speckle contrast imaging 


\section{Abstract}

Background: Changes in microvascular perfusion in scalds in children during the first four days, measured with laser speckle contrast imaging (LSCI), are related to the time to healing and need for surgical intervention. The aim of this study was to determine the accuracy (sensitivity and specificity) of LSCI on different days after injury in the prediction of healing outcome and if the accuracy can be improved by combining an early and a late measurement. Also, the accuracy of LSCI was compared with that of clinical assessment.

Methods: Perfusion was measured between 0-24 hours and between 72-96 hours using LSCI in 45 children with scalds. On the same occasions, burn surgeons assessed the burns as healing $<14$ days or healing >14 days/surgery. Receiver operating characteristic (ROC) curves were constructed for the early and late measurement and for the double measurement (DM) using two different methods.

Results: Sensitivity and specificity were 92.3\% (95\% CI: 64.0-99.8\%) and 78.3\% (95\% CI: 69.985.3\%) between 0-24 h, 100\% (95\% CI: 84.6-100\%) and 90.4\% (95\% CI: 83.8-94.9\%) between 72-96 h, and was 100\% (95\% CI: 59.0-100\%) and 100\% (95\% CI: 95.1-100\%) when combining the two measurements into a modified perfusion trend. Clinical assessment had an accuracy of $67 \%$, Cohen's $\kappa=0.23$.

Conclusion: The perfusion in scalds between 72-96 hours after injury, as measured using LSCI, is highly predictive of healing outcome in scalds when measured. The predictive value can be further improved by incorporating an early perfusion measurement within 24 hours after injury. 


\section{Introduction}

Assessment of partial-thickness burns is a difficult task even for the experienced burn surgeon, and the accuracy of assessment of burn depth varies between $60 \%$ and $75 \%$ [1]. Burn depth is usually assessed by investigating capillary refill time, sensibility and the appearance of the wound [2, 3]. These factors are all evaluated in a subjective way, often without the use of an established protocol. The accuracy therefore ultimately depends on the accumulated experience of the burn surgeon, and delays in surgical decisions are common. This may have implications for the patients, as it is known that early surgical treatment of deep dermal burns has a better clinical outcome with reduced prevalence of hypertrophic scarring [4, 5]. Early, accurate assessment of the burn is thus important for the patient because it allows for early intervention, if needed.

Many burns become deeper over time in a process called burn wound conversion [6-8]. The underlying mechanisms are not completely known, but local effects on the microcirculation with vasodilation, vasoconstriction and microthrombosis have been suggested to be of importance [6, 7]. Burn wound conversion is reported to occur early, often within the first 48 hours after injury $[7,8]$. Changes in microvascular measures that occur within a three-day time frame after injury, may therefore have high predictive value for the outcome of the wound.

Objective measurement of perfusion has been shown to be a reliable predictor of the healing capacity of the wound [9]. Laser Doppler Imaging (LDI) is currently the leading noninvasive method of measuring tissue perfusion in burns [10]. A study investigating the accuracy of clinical assessment by senior surgeons compared to LDI showed that the accuracy of the surgeons varied between $40.6 \%$ and $61.5 \%$ while LDI-assistance resulted in an accuracy of 5495\% during the first 0-3 days after injury [11]. On day 5, clinical assessment had 71.4\% accuracy 
while LDI had 97\% accuracy [11]. At 8 days after injury, however, both methods achieved 100\% accuracy indicating that the strength of the LDI technique lies in early predictions of burns [11]. The fact that LDI is so successful in early prediction of the wound outcome highlights the value of perfusion measurements for early assessment of burns as shown in several studies [11-21].

Despite the fact that LDI has been extensively validated, the technique has gained limited clinical use. This may be partly due to its cumbersomeness, long measurement time (a typical LDI scan takes 1-5 minutes to acquire and several scans may be needed) or issues related to motion artifacts. The risk for motion artifacts is particularly relevant in children. Some previous studies on LDI have therefore been confined to adults or adolescent patients [14, 16]. The recent development of a full-field laser Doppler imaging [22] is promising in that it reduces measurement time but it has yet to be proven in a clinical setting.

Laser speckle contrast imaging (LSCI), similar to LDI, constructs a color-coded map of the investigated area from dark blue to green, yellow and red showing low and high perfusion regions, respectively. Perfusion is measured in arbitrarily defined perfusion units (PU) [23]. Although both LDI and LSCI use laser light to estimate perfusion, the principle of measurement differs between the techniques. In LSCI, a divergent laser beam illuminates a rectangular area of tissue, typically $20 \times 20 \mathrm{~cm}$. In this area, a speckle pattern arises as a result of constructive and destructive interference of laser light [24, 25]. When the light is scattered off moving red blood cells, spatial and temporal fluctuations in the speckle pattern arise [24, 25]. Several image frames are acquired of the speckle pattern, and any fluctuations in the pattern causes blurring in the image frames [23-25]. The degree of blurring is quantified and is proportional to perfusion in the tissue [23-25]. The final image is a computed by calculating the mean of multiple individual 
frames, and total acquisition time ranges from a fraction of a second to a few seconds, depending on the required image quality [25].

We have previously described the complex temporal dynamics in perfusion seen in scalds depending on the depth of the burn [26]. However, the accuracy of LSCI in predicting burn healing time has not been studied. The primary aim of this study was to determine the accuracy (sensitivity and specificity), as well as the positive predictive value (PPV) and negative predictive value (NPV) of LSCI in the assessment of the healing time of scalds. We hypothesized that at specific measurement times, specific perfusion values can be used as cut-off values to predict the need for surgery. We also hypothesized that a greater sensitivity and specificity can be achieved when perfusion is measured at two occasions; one early measurement between 0 and 24 hours after injury, and one between 72 and 96 hours after injury. Furthermore, we compared the accuracy of LSCI with the accuracy of clinical assessments performed by experienced burn surgeons. 


\section{Methods}

\section{Study population}

Perfusion measurements were done using LSCI in pre-pubertal children with scalds who were admitted to the intensive burn care unit at Linköping University Hospital, or who were treated as outpatients in the same clinic. Patients were only included if a perfusion measurement had been made at either 0-24 hours or 72-96 hours after injury, or both. This requirement resulted in a total of 45 patients (19 of whom were female). Their mean age was 2.2 (range $0.8-8.6$ ) years and none of the patients had any underlying medical condition.

All patients initially received conservative treatment and a formal surgery decision was not made until 14 days after injury in accordance with local clinical practice. Surgery was done when the treating surgeon predicted that a wound area would take 21 days or more to heal. The same surgical procedure was used for all cases: sharp excision and autologous grafting of split thickness skin, usually meshed. Because all the children were under the age of consent, their parents or guardians gave permission for their participation in the study. The study was done in accordance with the Helsinki Declaration. The regional ethics committee approved the study on 22 February 2012, DNr 2012/31/31.

\section{Equipment}

We used a laser speckle contrast imager (Pericam PSI, Perimed AB, Järfälla, Sweden) to measure perfusion. The system uses a divergent laser beam with a wavelength of $785 \mathrm{~nm}$ to illuminate the tissue, and creates a speckle pattern in the area that is illuminated. It uses two cameras, one that captures the speckle contrast image and the other that captures a conventional color image of the 
measured area. The principle of the technique of LSCI has been previously described in detail [27].

We set the image size to correspond to a $12 \times 12 \mathrm{~cm}$ area of skin, kept the distance between the camera and the skin between 18 and $27 \mathrm{~cm}$, and set the acquisition rate to 21 frames/second. With each measurement, the system calculated the mean perfusion from 42 consecutive images, which resulted in a measurement time of 2 seconds for each scan. The spatial resolution of the perfusion image was $0.2 \mathrm{~mm} /$ pixel. The LSCI system was calibrated at regular intervals as recommended by the manufacturer.

\section{Measurement of perfusion}

All patients received sedatives before mechanical debridement and dressing of the wound(s), in accordance with normal clinical practice. Usually this was a combination of midazolam and ketamine, with the occasional addition of nitrous oxide. Propofol was used in cases with more extensive scalds. In a few cases, mostly older patients, satisfactory sedation was achieved by nitrous oxide alone.

During the first visit, which was between 6 and 72 hours after the injury, we always measured the perfusion in the wound directly after debridement and cleaning of the wound with sterile saline. In most patients we then made additional measurements each time the wound was dressed, typically every 2-4 days depending on the extent of the injury. Whenever possible we acquired images from the same areas of skin during subsequent visits so that we could follow changes in perfusion in the same wound over time. In the current study, only the perfusion data from the first, the third and the fourth day after injury were included, and perfusion 
measurements were categorized as either early ( $<24$ hours after injury) or late (72-96 hours after injury).

The standard dressing used was Mepilex AG (Mölnlycke, Health Care AB, Gothenburg, Sweden) and near full epithelialization Mepilex (Mölnlycke, Health Care AB, Gothenburg, Sweden) was sometimes used at the discretion of the responsible nurse. These dressings were always removed before image acquisition. Some wounds were covered by biological dressings or porcine xenografts (EZ Derm, Mölnlycke, Health Care AB, Gothenburg, Sweden), and those were not always removed when the wound was dressed. Biological dressings were used in 13 of the total 45 patients. As we have previously noticed that the presence of these dressings significantly reduces the measured perfusion, we excluded those regions from the analysis. We also excluded injuries on the hands (palmar), feet (plantar), and face, because we think that they may confound the data as a result of a relatively high and more variable baseline perfusion.

Regions of interest (ROI) were outlined in each image using the system's software (PIMSoft 1.5 Perimed AB, Järfälla, Sweden). Several images were usually acquired from the same patient, and each image could have multiple ROI in case there were wound regions that clearly had different perfusion. ROI were marked so that all different homogenous perfusion areas with a diameter of at least $2 \mathrm{~cm}$ were represented. When several areas had similar perfusion, but in different anatomical sites often with uninjured skin in between, each area contributed with one ROI. For each ROI, the mean perfusion was calculated and each ROI was outline by the first author (RM). The healing time for each ROI was defined as the number of days needed for complete (100\%) reepithelialization.

\section{Predicting surgery using LSCI}


To assess the accuracy of LSCI; true outcome was categorized as clinical healing of $<21$ days (negative) or $>21$ days/surgery (positive). Receiver operator characteristics (ROC) curves were calculated by using all available perfusion data from the early measurement and the late measurement. Cut-off values for maximal accuracy were calculated using Youden index. For the early measurement, two additional cut-off values were calculated. The first was chosen such that a sensitivity of $100 \%$ was achieved. The other cut-off was chosen based on the mean perfusion + 3 standard deviations of all wounds that healed after 21 days or which were treated by surgery $\left(p_{\mu \text {, surgery }}+3 S D\right)$. Assuming a Gaussian distribution, this cut-off would miss only $0.1 \%$ of the surgery cases. Finally, a cut-off value was also calculated for when $100 \%$ specificity was achieved for the late perfusion measurement.

To investigate if the change in perfusion over time was more predictive of the healing potential than single measurement, two additional assessment methods based on perfusion trend were investigated and their ROC curves calculated. The perfusion change $(\Delta P)$ over time between the first and fourth day after injury was calculated, where the early measurement always was done within 24 hours $\left(p_{1}\right)$ after injury and the late measurement between 72 and 96 hours after injury $\left(p_{2}\right)$. For simplicity, the time between the early and late measurement was always assumed to be 72 hours:

$\Delta P=\frac{p_{2}-p_{1}}{72}$

Since burn regions with the same perfusion change might have different initial perfusion levels, this has to be accounted for in the calculation of the perfusion trend, $\Delta P$. Otherwise, two different cases with the same perfusion change, but different initial values, would be treated the same according to the formula leading to an increased risk for false positive results. Therefore, we modified the method by adding a term that effectively puts more weight on initial perfusion, 
by increasing $\Delta P$ if the early perfusion was larger than the average early perfusion of all regions that needed surgery $\left(p_{\mu}\right.$, surgery $)$ :

$p_{1}<p_{\mu, \text { surgery }}+3 S D \rightarrow \Delta P_{\text {mod }}=\frac{p_{2}-p_{1}}{72}+\frac{p_{1}}{p_{\mu, \text { surgery }}}-1$

With this modified perfusion trend method, $\Delta P_{\text {mod }}$, regions were always considered to be negative (healing within 21 days) when $p_{1}>p_{\mu \text {, surgery }}+3 S D$.

\section{Discriminating between < II a (Partial thickness inj ury) and II b (full thickness injury) burns: accuracy using LSCI compared to clinical assessment}

During the first visit at the burn clinic the technician operating the LSCI system asked the burn surgeon on call to assess the different areas of the burn to discriminating between II a and II b burns, choosing between: 1) /II a) heals within 14 days, 2) II b) heals after 14 days or surgery required. The areas assessed and the different options were recorded on paper or digitally using annotated photographs. The burn surgeon then verified that the correct areas had been highlighted. This process was unrelated to the marking of ROI, which was done at a later time without detailed knowledge of the burn surgeon's assessment.

The accuracy of both the clinical assessment as well as LSCI was compared to that of the actual outcome for all patients who underwent clinical assessment and perfusion measurement 34 days after injury. In this comparison, the late perfusion measurement between 72 and 96 hours after injury $\left(p_{2}\right)$ was always used. Since the question to the surgeons was formulated as healing within 14 days or beyond, a different cut-off was calculated for the LSCI assessment using a separate ROC analysis and Youden index. Thus, this LSCI assessment was aimed at discriminating II a from II b burns instead of predicting surgical need. In addition to being below 
the cut-off at day 3-4, a hyperemic ring also had to be present for the ROI to be classified as healing $>14$ days based on the LSCI-measurement.

\section{Statistical analysis}

Within group tests were performed to check for differences in mean perfusion between 0-14 and 15-24 hours after injury and 72 and 96 hours after injury for the different severity strata using one-way analysis of variance with Sidak's correction for multiple comparisons. Kruskal-Wallis analysis with Dunn's correction for multiple comparisons was used to detect any differences in age among different burn severity strata. Correlation between clinical assessment and LSCI compared to true outcome was performed using Cohen’s $\kappa$.

Data are expressed as mean $( \pm \mathrm{SD})$ and the alpha level was set to 0.05 . All statistical analyses were made with the aid of GraphPad Prism version 5.02 for Windows (GraphPad Software, San Diego California USA, www.graphpad.com) and Excel 2013 (Microsoft, Redmond Washington USA, www.microsoft.com).

\section{Results}

There was no significant difference in age between regions of different severity (Table 1).

A total of 195 ROI were outlined in 45 patients, 129 ROI were available for analysis within 24 hours after injury and 147 ROI between 72 and 96 hours after injury. 81 ROI contained data from both time intervals and could be used for assessments based on the (modified) perfusion trend. Within-group tests showed no significant differences between perfusion values 
measured at different time points within 24 hours or for the mean perfusion between 72 and 96 hours after injury.

Receiver operator characteristics based on the early measurement $\left(p_{1}\right)$, the late measurement $\left(p_{2}\right)$, the perfusion trend $(\Delta P)$ and the modified perfusion trend $\left(\Delta P_{\text {mod }}\right)$ are shown in Figure 1. The resulting sensitivity, specificity, positive predictive value (PPV) and negative predictive value (NPV) with respective cut-off values are given in Table 2 . The mean perfusion within 24 hours in regions that needed surgery, $\left(p_{\mu}\right.$, surgery $)$, was $142 \mathrm{PU}$, with a standard deviation of $33 \mathrm{PU}$. Thus $p_{\mu}$, surgery $+3 S D$ was $241 \mathrm{PU}$. To further illustrate the effect of the absolute perfusion values and the perfusion trend between different days after injury on the outcome, perfusion data for each ROI is shown as a scatter diagram in Figure 2. The cut-off values for when surgery is indicated are also shown in Figure 2.

In 30 patients, both a detailed clinical assessment performed during the first visit, as well as perfusion data from the 72-96-hour interval after injury were available (33 ROI). Of all clinical assessments, 17 were done within 24 hours and 16 were done between 72-96 hours after injury. ROC analysis of the perfusion data revealed a cut-off of 180 PU to predict healing within 14 days or after 14 days, and this cut-off value was used in the comparison of accuracies of clinical assessment and LSCI. Using this cut-off, a $\kappa$ of 0.73 was found for LSCI assessment whereas clinical assessment yielded a $\kappa$ of 0.23 , which corresponds to moderate and minimal agreement, respectively [28]. The required presence of a hyperemic ring in all intermediate to deeper burns led to the correction of an otherwise false positive LSCI prediction in 1 out of the 33 ROI. 


\section{Discussion}

Our main finding was that the accuracy of early burn assessment can be greatly improved when assisted by LSCI. The accuracy depends on when the perfusion is measured, 3-4 days after injury being the best time-frame if a single measurement is done, with an accuracy of $91.8 \%$ (sensitivity $100 \%$, specificity $90.4 \%$ ). This level of accuracy is comparable to that reported in several studies using LDI [11-21]. However, when an additional perfusion measurement is done within the first 24 hours, the accuracy can be improved even further.

No statistically significant differences were found between perfusion measured between 72 and 96 hours after injury. Nor were any differences noted between perfusion measurements at different times within the first 24 hours after injury, regardless of burn severity. This suggests that the exact timing of the perfusion measurement is not critical, as long as it is done within the specified time frames.

The formulas used when calculating perfusion change based on an early and late measurement assumed a 72-hour time interval between the measurements, whereas the actual time interval varied between 48 and 90 hours. We think that this simplification was of no consequence in the present study, as there seems to be little change in perfusion during the first 24 hours or between 72-96 hours. Because of these observations it is reasonable to assume that temporal perfusion changes do not occur linearly, and that perfusion changes in the interval between 24 and 72 hours are of greater importance.

The assessment method solely based on the change in perfusion from 0-24 hours to 7296 hours better separated surgical cases from those that healed spontaneously when compared to single measurements. There were however still a few inaccuracies. For example, this method 
sometimes mistook very superficial burns, healing within 4 days, for deep burns that required surgery. This prompted a modification to the algorithm in which the initial perfusion was given more weight and in which additional measurements were discarded when $p_{1}$ had a perfusion value 3 standard deviations above the mean perfusion value for wounds that require surgery. By doing this, only $0.1 \%$ of the wounds requiring surgery would go undetected by the early measurement, assuming the perfusion follows a Gaussian distribution. With these two modifications, the number of false positives was reduced to zero, while still retaining the same sensitivity.

A single perfusion measurement using LSCI after 72 to 96 hours after injury results in a high NPV, reaching $100 \%$ with a PPV of $63.6 \%$. This means that the technique can be used to identify the majority of the wound regions (90.4\%) which will not have to undergo surgery after 72 to 96 hours after injury. If an early measurement is also made within the first 24 hours after injury, the two measurements combined allow for an early prediction of the need for surgery with up to $100 \%$ accuracy. This strong predictive value may be used to facilitate a surgical decision, and allows surgical intervention as early as 3-4 days after injury.

It is important to note that clinical decision making still rests upon the experience of the burn surgeon. LSCI should not be used for scald burns that are either clearly full-thickness or superficial. The technique should instead be used to support decision making for the many unclear cases. Based on the findings in this study, we suggest an approach based on clinical evaluation, with additional perfusion measurements, preferably done within 24 hours after injury. If the perfusion is >240 PU during the first 24 hours, the future need of surgical intervention can be ruled out with a high probability; if not, another measurement can be done 72-96 hours after the injury and with the aid of these two measurements surgical need can be predicted. 
In cases where there only is one available measurement 3-4 days after injury, a value $>170$ PU can be considered to safely rule out the need of surgery, while a value $<130 \mathrm{PU}$ indicates a clear need of surgery. These two cut-off values correspond to when $100 \%$ sensitivity and $100 \%$ specificity was achieved, respectively. With perfusion values between 130-170 PU, predictions cannot reliably be made and active expectation might be the best option until a clear diagnosis can be achieved. Also, measurements done within the first 24 hours after injury should not be the basis for a positive decision of surgical intervention alone. An early measurement should only be used to exclude surgical need.

LSCI has some minor technical limitations. Artifacts are sometimes generated as a result of specular reflections that are not completely blocked by the polarization filter in front of the camera lens. These present as areas of pixels with very low or even negative perfusion values, and seem to occur most frequently on moist wound surfaces and when the area of interest is curved. Fortunately, as long as the polarization filter is mounted correctly in relation to the polarization direction of the laser, these artifacts are easily recognized and are seldom an issue. Movement artifacts can also in some situations be a source of error, especially in unsedated children. Any movement of the patient during the image acquisition will increase the measured perfusion values. There are several ways to mitigate the effects of patient motion. One possibility is to use a movement filter [29] or by acquiring images of the same area in rapid succession, and choosing the image with the lowest perfusion value [30]. However, it is our experience that accurate LSCI measurement can be done in children in a setting of minimal to moderate sedation, which may not be possible using LDI.

Education for both the technicians using the system, and also for the burn surgeons should be considered for adequate interpretation of the perfusion images. Also, optimal implementation 
of LSCI assessment into the clinical practice may be difficult since measurements both at 0-24 hours and 72-96 hours after injury are needed to maximize the predictive value. Nonetheless, a single measurement between 72 and 96 hours could improve burn assessments and facilitate early surgery and could be implemented in the clinical workflow relatively easily. The value of early surgery, based on data from the LSCI measurements, must also be evaluated in a prospective RCT before introduction to clinical practice.

Another factor to consider when using LSCI to assist clinical decision making is whether a hyperemic ring is surrounding the ROI. If a zone of stasis is present, there should be a hyperemic ring surrounding it. When this is not the case the burn was likely not severe enough to cause a maximal vasodilatory response. This phenomenon can be seen in Figure 3, showing a burn region with a central area of intermediate depth healing in 10 days surrounded by a hyperemic zone, as well as a region with the same perfusion level, which instead healed within 4 days. A deeper burn, corresponding to the zone of stasis, should always be surrounded by a hyperemic ring and when this is not present, one should presume that the burn is superficial regardless of the perfusion value measured.

Yet another factor of importance is the area of the burn region being investigated, especially with regards to the maximal distance to hyperemic skin. A low-perfusion region with an initial diameter of roughly $1 \mathrm{~cm}$ may suggest surgical need, but usually recovers spontaneously. Such areas were observed, but did not fulfill the ROI marking criteria and were not common enough to make a conclusive analysis of the phenomenon. Therefore, care should be taken with assessment of perfusion in regions of limited size. At the same time, these areas are generally too small to warrant surgical intervention. 
In the second part of the study, which investigated the accuracy of clinical assessment and LSCI in discrimination of II a from II b burns, it was necessary to use different cut-off values than the perfusion-based methods for predicting surgical need. It seems like LSCI has a lower predictive value, using a single measurement after 72 to 96 hours, in separating wounds healing within 14 days from those healing after 14 days/surgery, while it has excellent accuracy in separating wounds healing within 21 days from those requiring surgery. For LSCI to more exactly predict the healing time in wounds healing within 21 days, a method based on the perfusion trend likely has to be used. Additionally, 17 of the 33 clinical assessments were done within 24 hours after injury whereas all LSCI assessments were based on data captured at 72 to 96 hours. Sub-category analysis of clinical assessments done at 72-96 hours after injury shows that these had an accuracy of $62.5 \%$ and $\kappa$ of 0.25 while the total accuracy for clinical assessment was $66.7 \%$ and a $\kappa$ of 0.23 . It thus seems reasonable to assume that merging of clinical assessments within 24 hours and at 72 to 96 hours after injury would have little effect on the result.

Most studies investigating objective methods for burn assessment use accuracy to evaluate the feasibility of the different methods. A limitation of accuracy is that it does not take into account the probability of chance agreement, which can be of consequence at lower accuracies [28]. We therefore used Cohen's kappa to more exactly estimate the true accuracy when comparing clinical assessment with assessment using LSCI.

A strength of the study is that surgical intervention was always delayed for at least 14 days after injury, according to local clinical practice, meaning that all burns regarded as deep partial-thickness were observed long enough to guarantee correct depth assessment. This is an important aspect since no histology was used to otherwise confirm burn depth. This study 
otherwise has several limitations. All ROI were outlined by the same observer, which could be a source of bias. Therefore, it is important to investigate the interrater reliability of LSCI measurements in the future. Additionally, only scald burns were investigated and most wounds were confined to the upper part of the torso, arms and thighs. All palmar, plantar and facial wounds were excluded since our preliminary findings indicate that these may have different perfusion dynamics and require different values and measurement times to successfully be evaluated. Future studies are needed to investigate how these areas should be assessed using LSCI.

Furthermore, only prepubertal children were included in the study. Dermal thickness increases with age and is influenced by puberty for both genders [31, 32]. Testosterone has been shown to increase epidermal thickness and alter the constituents of dermis, especially in males [33, 34]. Additionally, estrogen has vasodilatory actions [35] and likely increases basal perfusion, which might in turn affect the hyperemic response in burns. This may result in a sex-related effect on healing time and measured perfusion [20]. Therefore, the results presented in this study should not be applied to an adolescent or adult population. Although the general principles described in this study may apply, the cut-off values could be different. 


\section{Conclusion}

In conclusion, our results indicate that LSCI should be considered a valuable tool for accurately predicting surgical need in scald burns in prepubertal children. The early perfusion dynamics in scald burns in children can be used to even further increase the predictive value within 3-4 days after injury. Perfusion measurements using LSCI within 24 hours after injury have limited positive predictive value but a high negative predictive value and should therefore only be used to exclude the need for surgery. 


\section{References}

[1] Devgan L. et al. Modalities for the assessment of burn wound depth. J Burns Wounds. 2006 Feb 15;5:e2. doi: 10.1016/j.burns.2008.01.009.

[2] Kim L.K. et al. Medical management of paediatric burn injuries: Best practice. J Paediatr Child Health. 2012 Apr;48(4):290-5. doi: 10.1111/j.1440-1754.2011.02128.x.

[3] Monstrey S. et al. Assessment of burn depth and burn wound healing potential. Burns. 2008 Sep;34(6):761-9. doi: 10.1016/j.burns.2008.01.009.

[4] Tredget E. et al. Biology and Principles of Scar Management and Burn Reconstruction. Surg Clin North Am. 2014 Aug;94(4):793-815. doi: 10.1016/j.suc.2014.05.005.

[5] Cubison T.C. et al. Evidence for the link between healing time and the development of hypertrophic scars (HTS) in paediatric burns due to scald injury. Burns. 2006 Dec;32(8):992-9. doi: 10.1016/j.burns.2006.02.007

[6] Singh V. et al. The pathogenesis of burn wound conversion. Ann Plast Surg. 2007 Jul;59(1):109-15. doi: 10.1097/01.sap.0000252065.90759.e6

[7] Salibian A.A. et al. Current concepts on burn wound conversion-A review of recent advances in understanding the secondary progressions of burns. Burns. 2016 Aug;42(5):1025-35. doi: 10.1016/j.burns.2015.11.007.

[8] Yang Z. (ed). Phenomenon of Progressive Deepening in Burn Wounds. In: Chinese burn surgery. Springer Netherlands; 2015. p. 210. doi: 10.1007/978-94-017-8575-4 
[9] Thatcher J.E. et al. Imaging Techniques for Clinical Burn Assessment with a Focus on Multispectral Imaging. Adv Wound Care (New Rochelle). 2016 Aug 1;5(8):360-378. doi: 10.1089/wound.2015.0684

[10] Kaiser M. et al. Noninvasive assessment of burn wound severity using optical technology: A review of current and future modalities. Burns. 2011 May;37(3):377-86. doi: 10.1016/j.burns.2010.11.012.

[11] Hoeksema H. et al. Accuracy of early burn depth assessment by laser Doppler imaging on different days post burn. Burns. 2009 Feb;35(1):36-45. doi: 10.1016/j.burns.2008.08.011.

[12] La Hei E.R. et al. Laser Doppler Imaging of paediatric burns: Burn wound outcome can be predicted independent of clinical examination. Burns. 2006 Aug;32(5):550-3. doi: 10.1016/j.burns.2005.11.014

[13] Cho J.K. et al. Relationship between healing time and mean perfusion units of laser Doppler imaging (LDI) in pediatric burns. Burns. 2009 Sep;35(6):818-23. doi:

10.1016/j.burns.2008.12.009.

[14] Burke-Smith A. et al. A comparison of non-invasive imaging modalities: Infrared thermography, spectrophotometric intracutaneous analysis and laser Doppler imaging for the assessment of adult burns. Burns. 2015 Dec;41(8):1695-707. doi: 10.1016/j.burns.2015.06.023.

[15] Hoeksema H. et al. A new, fast LDI for assessment of burns: a multi-centre clinical evaluation. Burns. 2014 Nov;40(7):1274-82. doi: 10.1016/j.burns.2014.04.024.

[16] McGill D.J. et al. Assessment of burn depth: a prospective, blinded comparison of laser Doppler imaging and videomicroscopy. Burns. 2007 Nov;33(7):833-42. 
[17] Erba P. et al. FluxEXPLORER: a new high-speed laser Doppler imaging system for the assessment of burn injuries. Skin Res Technol. 2012 Nov;18(4):456-61. doi: 10.1111/j.16000846.2011.00593.x.

[18] Holland A.J. et al. Laser Doppler imaging prediction of burn wound outcome in children. Burns. 2002 Feb;28(1):11-7.

[19] Stewart T.L. et al. The use of laser Doppler imaging as a predictor of burn depth and hypertrophic scar postburn injury. J Burn Care Res. 2012 Nov-Dec;33(6):764-71. doi: 10.1097/BCR.0b013e318257db36.

[20] Baker R.D. et al. Using ordinal logistic regression to evaluate the performance of laserDoppler predictions of burn-healing time. BMC Med Res Methodol. 2009 Feb 16;9:11. doi: 10.1186/1471-2288-9-11.

[21] Shin J.Y, Yi H.S. Diagnostic accuracy of laser Doppler imaging in burn depth assessment: Systematic review and meta-analysis. Burns. 2016 Nov;42(7):1369-1376. doi: 10.1016/j.burns.2016.03.012.

[22] van Herpt H. et al. Burn imaging with a whole field laser Doppler perfusion imager based on a CMOS imaging array. Burns 2010 May;36(3):389-96. doi: 10.1016/j.burns.2009.05.009.

[23] Briers J.D. Laser Doppler, speckle and related techniques for blood perfusion mapping and imaging. Physiol. Meas. 2001 Nov;22(4):R35-66.

[24] Boas D. A, Dunn A.K. Laser speckle contrast imaging in biomedical optics. J Biomed Opt. 2010 Jan-Feb;15(1):011109. doi: 10.1117/1.3285504 
[25] Basak, K. et al. Review of laser speckle-based analysis in medical imaging. Med Biol Eng Comput. 2012 Jun;50(6):547-58. doi:10.1007/s11517-012-0902-z.

[26] Mirdell R, Iredahl F, Sjöberg F, Farnebo S, Tesselaar E. Microvascular blood flow in scalds in children and its relation to duration of wound healing: A study using laser speckle contrast imaging. Burns. 2016 May;42(3):648-54. doi: 10.1016/j.burns.2015.12.005.

[27] Lindahl F, Tesselaar E, Sjöberg F. Assessing paediatric scald injuries using laser speckle contrast imaging. Burns. 2013;39:662-6. doi: 10.1016/j.burns.2012.09.018.

[28] McHugh M.L. Interrater reliability: the kappa statistic. Biochem Med (Zagreb). 2012 Oct; 22(3): 276-282.

[29] Omarjee L. et al. Optimisation of movement detection and artifact removal during laser speckle contrast imaging. Microvasc Res. 2015 Jan;97:75-80. doi: 10.1016/j.mvr.2014.09.005.

[30] Zötterman J. et al. Methodological concerns with laser speckle contrast imaging in clinical evaluation of microcirculation. PLoS One. 2017 Mar 30;12(3):e0174703. doi: 10.1371/journal.pone.0174703.

[31] Seidenari S. et al. Thickness and Echogenicity of the Skin in Children as Assessed by 20MHz Ultrasound. Dermatology 2000;201:218-222. doi: 10.1159/000018491

[32] Derraik J.G. et al. Effects of Age, Gender, BMI, and Anatomical Site on Skin Thickness in Children and Adults with Diabetes. PLoS One. 2014; 9(1): e86637. doi:

10.1371/journal.pone.0086637

[33] Köhn F.M. Testosterone and body functions. The Aging Male. 2006 Dec;9(4):183-8. doi: $10.1080 / 13685530601060396$ 
[34] Shin M.H. et al. Modulation of Collagen Metabolism by the Topical Application of Dehydroepiandrosterone to Human Skin. J Invest Dermatol. 2005 Feb;124(2):315-23. doi: 10.1111/j.0022-202X.2004.23588.X

[35] White R.E. Estrogen and vascular function. Vascul Pharmacol. 2002 Feb;38(2):73-80. 


\begin{tabular}{|c|c|c|c|c|c|c|c|c|}
\hline & \multicolumn{2}{|c|}{ Number of } & \multirow[t]{2}{*}{$\begin{array}{c}\text { Age } \\
\text { (years) }\end{array}$} & \multirow[t]{2}{*}{ \%TBSA } & \multicolumn{3}{|c|}{ Average Perfusion (PU) at } & \multirow[b]{2}{*}{96 hours } \\
\hline & patients & $R O I$ & & & $\begin{array}{l}0-14 \\
\text { hours }\end{array}$ & 15-24 hours & 72 hours & \\
\hline \multicolumn{9}{|l|}{ Healed after } \\
\hline 3-4 days & 10 & 20 & $3.2 \pm 2.4$ & $5.2 \pm 3.1$ & $241 \pm 39$ & $303 \pm 68$ & $265 \pm 57$ & $217 \pm 82$ \\
\hline 5-7 days & 32 & 68 & $2.2 \pm 1.7$ & $5.1 \pm 3.8$ & $275 \pm 54$ & $283 \pm 65$ & $489 \pm 108$ & $440 \pm 91$ \\
\hline 8-11 days & 28 & 61 & $2.1 \pm 1.7$ & $5.1 \pm 3.6$ & $221 \pm 34$ & $211 \pm 63$ & $348 \pm 125$ & $422 \pm 123$ \\
\hline 12-18 days & 13 & 18 & $2.6 \pm 2.1$ & $5.0 \pm 2.0$ & $148 \pm 10$ & $149 \pm 20$ & $194 \pm 40$ & $196 \pm 78$ \\
\hline $\begin{array}{l}\text { Surgery } \\
\text { required }\end{array}$ & 10 & 28 & $2.3 \pm 1.6$ & $7.8 \pm 5.2$ & $137 \pm 26$ & $148 \pm 42$ & $98 \pm 29$ & $116 \pm 33$ \\
\hline Total & 45 & 195 & $2.2 \pm 1.6$ & $5.1 \pm 3.7$ & & & & \\
\hline
\end{tabular}

Table 1. The number of patients with scald burns included in the study, as well as the number of ROI, the mean \pm SD age of the patients at the time of injury, the mean \pm SD \%TBSA and the mean \pm SD perfusion in the wound regions, for wounds of different severity. No significant differences in perfusion were detected for the within group tests (0-14 vs. 15-24 hours and 72 vs. 96 hours) nor were any significant differences in age detected. 10 ROI in 10 patients healed within 14 days in the "Healed after 12-18 days" category. 


\begin{tabular}{|c|c|c|c|c|c|}
\hline Method & Cut-off & Sensitivity (95\% CI) & Specificity (95\% CI) & PPV & NPV \\
\hline 0-24h SM & $173 \mathrm{PU}$ & $92.3 \%$ (64.0-99.8\%) & 78.5\% (69.9-85.5\%) & $32.4 \%$ & $98.9 \%$ \\
\hline 0-24h SM & $210 \mathrm{PU}$ & $100 \%(75.3-100 \%)$ & $67.2 \%$ (57.9-75.7\%) & $25.5 \%$ & $100 \%$ \\
\hline 0-24h SM & $240 \mathrm{PU}$ & $100 \%(75.3-100 \%)$ & $53.5 \%(44.0-62.8 \%)$ & $19.4 \%$ & $100 \%$ \\
\hline 72-96h SM & $130 \mathrm{PU}$ & 72.7\% (49.8-89.3\%) & 100\% (97.1-100\%) & $100 \%$ & $95.4 \%$ \\
\hline 72-96h SM & $170 \mathrm{PU}$ & $100 \%(84.6-100 \%)$ & $90.4 \%$ (83.8-94.9\%) & $64.7 \%$ & $100 \%$ \\
\hline Trend DM & $-0.35 \Delta P$ & $100 \%$ (59.0-100\%) & 93.2\% (84.9-97.8\%) & $58.3 \%$ & $100 \%$ \\
\hline Mod trend DM & $-0.35 \Delta P_{\text {mod }}$ & $100 \%(59.0-100 \%)$ & $100 \%$ (95.1-100\%) & $100 \%$ & $100 \%$ \\
\hline
\end{tabular}

Table 2. Sensitivity, specificity, PPV (positive predictive value) and NPV (negative predictive value) for different cut-off values using different methods, SM (single measurement) and DM (double measurement), and at different time points after injury. For the sensitivity and specificity, the 95\% CI (confidence interval) is also shown. 


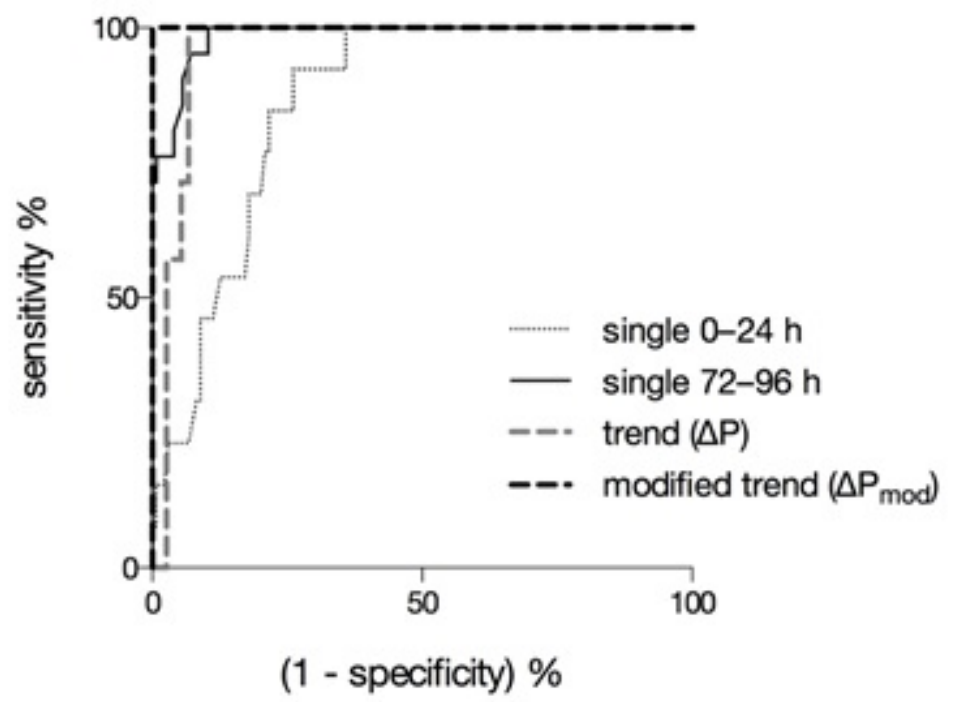

Figure 1. Accuracy of assessment of scalds using LSCI using four different methods, shown as ROC (receiver operating characteristics) curves with sensitivity on the y-axis and 1-specificity on the $\mathrm{x}$-axis. The curves show how the sensitivity and specificity varies for different perfusion cut-off values. The AUC and its $95 \% \mathrm{CI}$ for the four different methods: single $0-24 \mathrm{~h}$ was 0.90 (0.83-0.96), single $72-96 \mathrm{~h}$ was $0.98(0.97-1.00)$, trend $(\Delta \mathrm{P})$ was $0.96(0.91-1.00)$ and modified trend $\left(\Delta P_{\text {mod }}\right)$ was $1.00(1.00-1.00)$. 


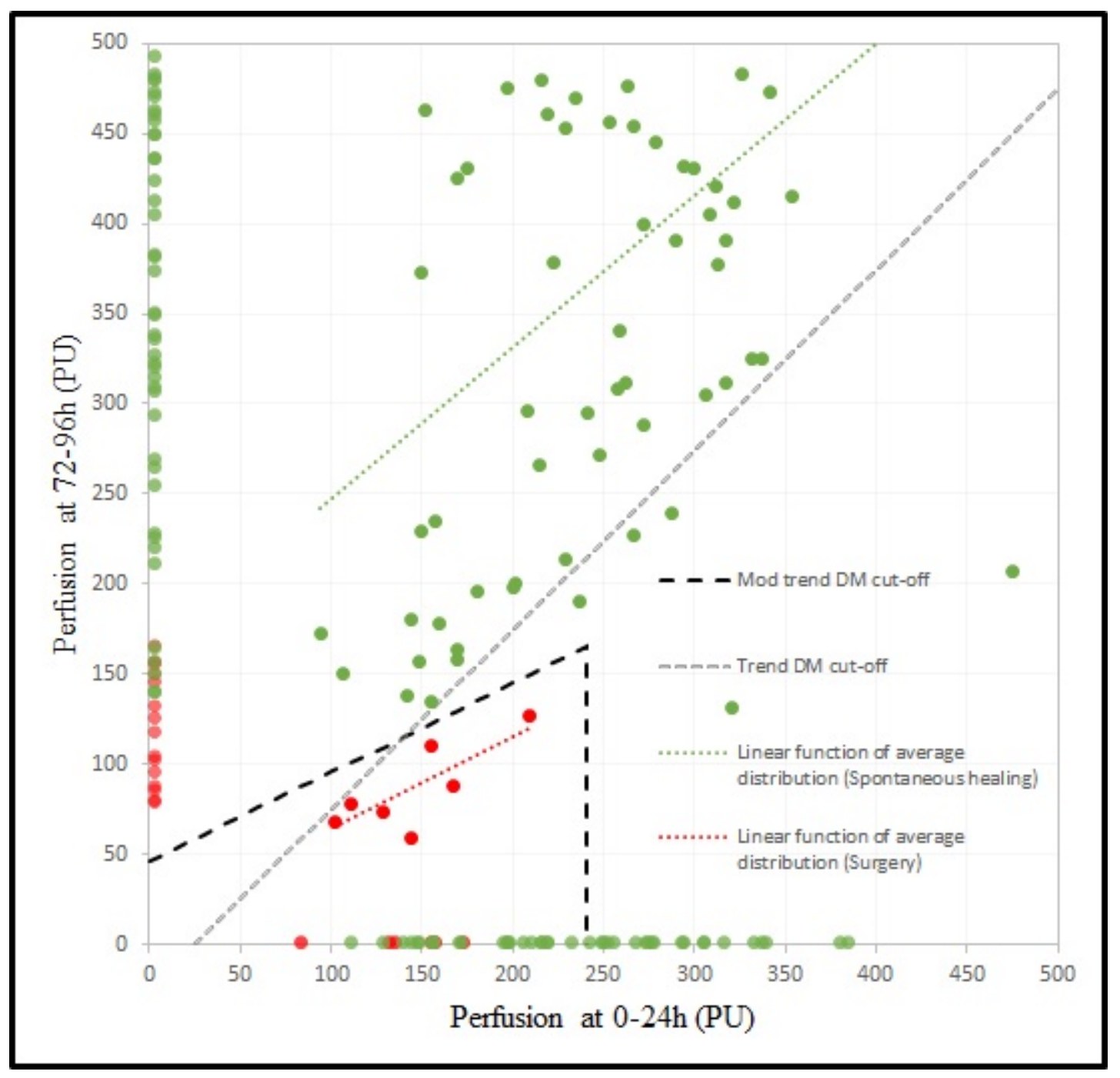

Figure 2. Scatter plot diagram where each green dot corresponds to a ROI that healed within 21 days and each red dot corresponds to a ROI requiring surgery. Perfusion, as measured using LSCI, 0-24 hours after injury is described on the x-axis and perfusion 72-96 hours after injury on the $y$-axis. Dots on the $\mathrm{x}$-axis indicates ROI for which only an early measurement was available, whereas for dots on the y-axis, only a late measurement was done. For all other dots, both early and late perfusion measurements were available. The dotted lines of green and red color correspond to a linear equation describing the average distribution. The grey dashed line represents a cut-off value of $-0.35 \Delta P$ using the simple trend method and the black dashed line represents the cut-off value of $-0.35 \Delta P_{\text {mod }}$ using the modified trend method. 


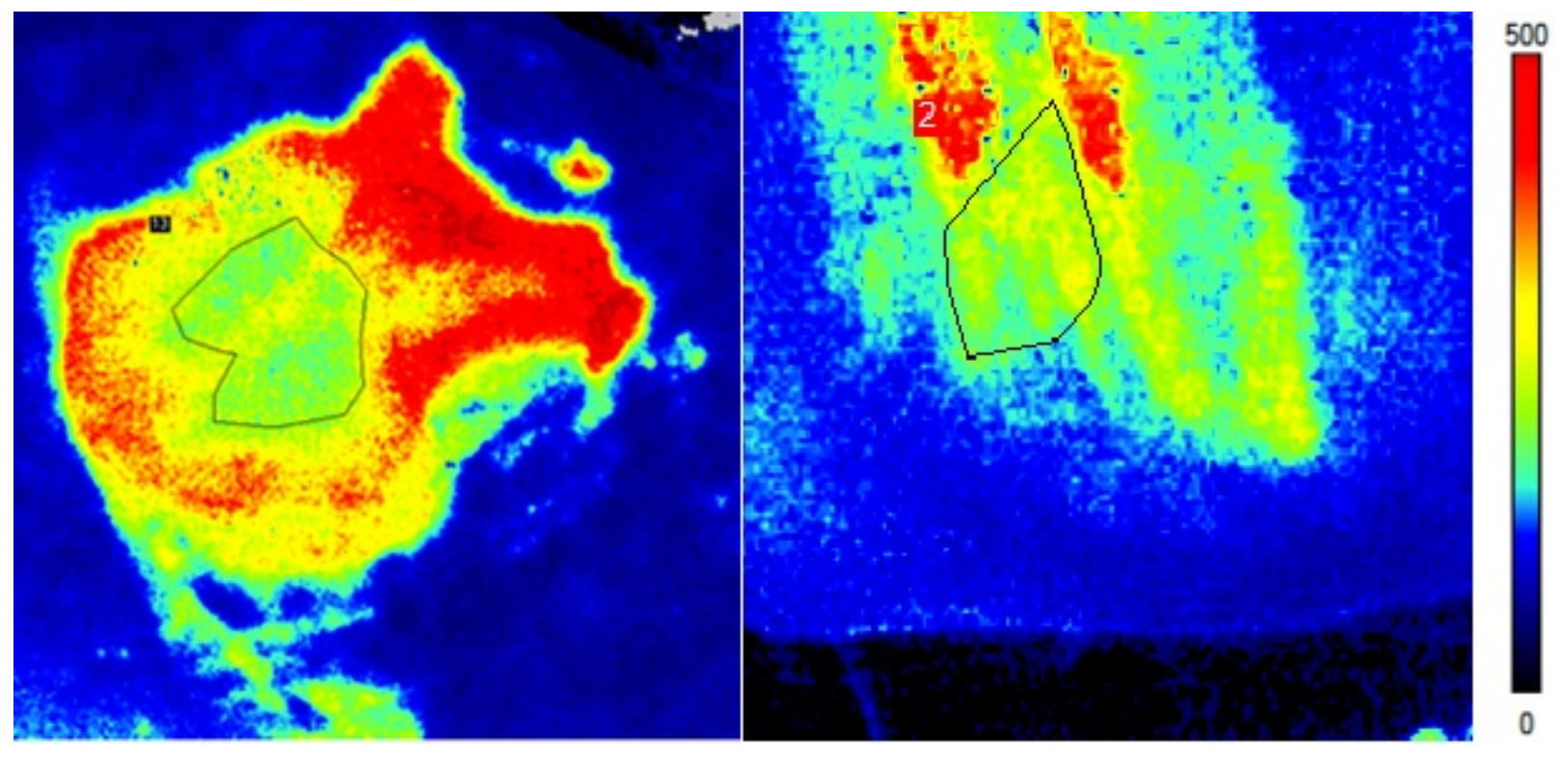

Figure 3. Scald burn in two different patients (left) 4 days and (right) 3 days after injury. Both ROI marked with a black line have the same perfusion, but the patient to the left shows a hyperemic ring surrounding the deeper burn in the middle, whereas the patient to the right shows no indication of the same phenomena and instead has a hyperemic zone creating no ring with a deeper central component. The left patient achieved spontaneous reepithelialization 10 days after injury while the right patient required 4 days. This illustrates that perfusion values should not be interpreted in a void and that the state of the surrounding skin can provide vital information. 